\title{
The potential of exploiting non-symmetric structures in electrical machines
}

\author{
Stefano Nuzzo, Alessandro Marfoli, Luca Papini, Paolo Bolognesi, Chris Gerada, Michael Galea \\ Power Electronics, Machines and Control Group \\ University of Nottingham \\ Nottingham, UK
}

\begin{abstract}
Typically, electrical machines feature rotor and stator winding configurations and core structures which comply with both simplicity and cost requirements. Such constraints limit the design search space and degrees of freedom, thus leading to exploit and implement only standard symmetrical layouts which therefore represent by far the most common choice. In this paper, the potential of adopting non-symmetrical windings and geometries in electrical machines is discussed. The perceived benefits are first detailed and then proven by applying the concepts to two case studies, namely a squirrel-cage induction machine and a salient-pole synchronous machine.
\end{abstract}

Index Terms-Asymmetries, damper cage, induction machine, non-symmetrical windings, power quality, synchronous generator, torque ripple.

\section{INTRODUCTION}

Symmetry is widely exploited in electrical machines aiming to minimize manufacturing costs and the design process complexity [1], [2]. Therefore, most commonly, symmetrical and regular stator and rotor structures are used [3], [4]. Nevertheless, this choice comes at the cost of reducing the design degrees of freedom which would be available if asymmetrical configurations were investigated. However, more stringent requirements on power density [5], reliability [6], [7] and power quality [8], [9], driven by environmental regulations and increased demand for high efficient electrical machines, is pushing towards the development of new materials, innovative manufacturing processes and alternative machine design topologies [10]-[16].

In this context, the adoption of asymmetrical windings and/or core structures represents an interesting alternative without necessarily compromising the cost and complexity minimization goals aforementioned. For example, a symmetrical multi-layer, fractional-slot, concentrated winding is proposed in [11], targeting a reduction of both the magnetomotive-force (MMF) harmonics and the eddy current losses in the permanent magnets (PMs). A stator topology where both the winding arrangement and the peripheral slot pattern are unconventional is presented in [12], showing significant improvements in terms of MMF waveform. In [10], an asymmetricallydisplaced damper cage is implemented onto a 4-MVA woundfield synchronous generator (SG) to reduce the relevant ohmic losses induced at no-load as well as at on-load operations. In [13], the same technique of tailoring the damping bars' position is applied to a smaller alternator to improve the voltage waveforms' quality, permitting to eliminate the need for stator slot skewing. In [14], [15], asymmetric rotor flux barriers are implemented to reduce the torque ripple in synchronous reluctance machines. In particular, the rotor structure reported in [14] features an even-symmetric design with respect to two pole pitches, i.e. the flux barriers' geometries relative to two adjacent poles are different to each other. The same concept is applied in [15], where asymmetric flux barriers are designed in such a way that the rotor presents an oddsymmetric arrangement with respect to one pole pitch. In [16], the cogging torque of an axial PM motor is reduced by employing different magnet spans.

These works prove that asymmetrical structures can produce a number of beneficial effects. However, these existing publications focus on specific platforms and optimization objectives, without providing a general framework and applicability of such powerful techniques. Therefore, the aim of this paper is to address this challenge and this is done according to the methodology described as follows. The theoretical background relative to the winding functions (WFs) characterizing the machine phases is recalled in Section II, whereas in Section III, the potential benefits of departing from standard configurations where symmetrical structures represent only a single and particular case are highlighted. The approach presented in these two sections is not referred to any specific case study and is thus aimed at proving the general applicability of the method. However, in order to prove the concepts, two practical case studies are then investigated, namely a squirrelcage induction machine (IM) and a salient-pole SG. Section IV details the positive effects of an irregular stator slot opening patterns on the torque ripple of an IM case study. Section $\mathrm{V}$ shows how the voltage total harmonic distortion (THD) can be reduced by asymmetrically displacing the damping bars onto the salient poles of a SG case study. Although in this paper the potential of using non-symmetrical structures is only demonstrated through unconventional patterns of the slot openings of an IM and of the damping bars of a SG, additional design parameters and different types of electrical machines can be exploited.

\section{BACKGROUND}

WFs have been widely used as tool for the electromagnetic design and analysis of electrical machines. During the design stage, they can turn out particularly useful for selecting a winding arrangement which complies with specific perfor- 
mance requirements. They are also extensively employed for the analysis of existing winding configurations, mainly aiming to evaluate key characteristics of electrical machines such as the MMFs and their harmonics, fluxes and inductances profiles [17]. Although the WF method is well-known, the theoretical background relative to the description of the machines' phases through WFs is recalled in this section, aiming at highlighting the implications of symmetrical and asymmetrical winding layouts.

To define the machine WFs, first it is assumed that the total number of phases is $m$ and that each of them consists of $n_{k}$ groups of active conductors, with $k=1, \ldots, m$. The position of such groups is assumed to be either $a$ ) in correspondence of rotor and stator slot openings for open or semi-closed structures or $b$ ) in correspondence of equivalent rotor and stator slot openings when a closed slot layout is adopted. In each group, the information relative to the equivalent position of such conductors, eventually related to the position of the opening of the slot hosting them, is given by $\lambda_{k, q}(\alpha)$, with $q=1, \ldots, n_{k}$. The parameter $\lambda$ is the normalized coordinate (associated to the rotor angular reference frame) mapping the whole airgap along the tangential direction by spanning the $[0,1)$ interval and $\alpha$ is the position of rotor vs. stator. The number of active conductors in each group is provided by the quantity $N_{k, q}$, which can feature either positive or negative values according to the convention defined for the current direction. Considering the above, it becomes possible to define the WF of any generic phase $k$ as in (1).

$$
N_{k}(\lambda, \alpha)=\sum_{q=1}^{n_{k}} N_{k, q} \cdot \delta\left(\lambda_{k, q}(\alpha), \lambda\right)
$$

In (1), the function $\delta\left(\lambda_{k, q}(\alpha), \lambda\right)$ is equal to 1 when the considered set $k, q$ of active conductors is within the closed loop delimited by an arbitrary fixed position $\lambda=0$ and by the generic position $\lambda$, otherwise it is equal to 0 . Having defined the WF as in (1), the next step is that of developing $N_{k}(\lambda, \alpha)$ using the Fourier series decomposition with respect to the variable $\lambda$. Assuming that the machine under study features $p$ pole pairs and focusing only on the absolute values of the amplitudes $\left|{ }^{h} N_{k}(\alpha)\right|$ of the Fourier harmonic components (where $h$ is the harmonic order), these can be written as in (2), where the complex form is adopted to facilitate the analytical elaboration.

$$
\left|{ }^{h} N_{k}(\alpha)\right|=2 \cdot\left|\frac{1}{1 / p} \cdot \int_{0}^{1 / p} N_{k}(\lambda, \alpha) \cdot e^{-j \frac{2 \pi}{1 / p} h \lambda} \cdot d \lambda\right|
$$

By substituting the right-hand side member of (1) in (2), the expression (3) is obtained. Then, by leveraging on the linearity property of the integral and considering that a complex number and its conjugate feature the same absolute value, (3) can be re-written as in (4).

$$
\begin{aligned}
& \left.\right|^{h} N_{k}(\alpha) \mid= \\
& 2 p\left|\int_{0}^{1 / p}\left[\sum_{q=1}^{n_{k}} N_{k, q} \cdot \delta\left(\lambda_{k, q}(\alpha), \lambda\right)\right] \cdot e^{-j \frac{2 \pi}{1 / p} h \lambda} \cdot d \lambda\right|
\end{aligned}
$$

$$
\begin{aligned}
& \left|{ }^{h} N_{k}(\alpha)\right|= \\
& 2 p \cdot\left|\sum_{q=1}^{n_{k}} N_{k, q} \cdot \int_{0}^{1 / p} e^{j \frac{2 \pi}{1 / p} h \lambda} \cdot \delta\left(\lambda_{k, q}(\alpha), \lambda\right) \cdot d \lambda\right|
\end{aligned}
$$

Carrying on with this mathematical survey aimed at finding a compact expression of $\left|{ }^{h} N_{k}(\alpha)\right|$, the definition of the function $\delta\left(\lambda_{k, q}(\alpha), \lambda\right)$ permits to modify the lower limit of the integration interval. In fact, recalling that $\delta\left(\lambda_{k, q}(\alpha), \lambda\right)=0$ when $\lambda \epsilon\left(0, \lambda_{k, q}\right)$, the lower limit 0 is replaced with $\lambda_{k, q}$ and $\delta\left(\lambda_{k, q}(\alpha), \lambda\right)$ is removed. These observations are implemented in (5).

$$
\left|{ }^{h} N_{k}(\alpha)\right|=2 p \cdot\left|\sum_{q=1}^{n_{k}} N_{k, q} \cdot \int_{\lambda_{k, q}}^{1 / p} e^{j \frac{2 \pi}{1 / p} h \lambda} \cdot d \lambda\right|
$$

The last steps consists in observing that only the groups of conductors whose equivalent position falls within the interval $\lambda_{k, q}$ provide some contribution to integral $(0,1 / p)$ and in solving the integral (5). Therefore, first the sub-set $\tilde{q}$ is introduced. This comprises all those $q$ indexes such that $\lambda_{k, q}(\alpha)$ belongs to the interval delimited by the positions $\lambda=0$ and $\lambda=1 / p$. Keeping in mind that the sum of $N_{k q}$ over $\tilde{q}$ is zero, the integral (5) can be manipulated to provide the following expression:

$$
\left|{ }^{h} N_{k}(\alpha)\right|=\frac{1}{\pi h}\left|\sum_{q \in \tilde{q}} N_{k q} \cdot e^{j 2 \pi p h \lambda_{k q}(\alpha)}\right|
$$

\section{EFFEcts of Asymmetric Structures ON WFs AND MMFS}

When no constraints are imposed on the slot pitch, on the coil pitch and on the number of conductors-per-coil, the quantities $N_{k, q}$ and $\lambda_{k, q}(\alpha)$ involved in (6) can assume any reasonable value, thus representing the whole set of degrees of freedom available during the design of the electrical machine. The exploitation of these degrees of freedom can thus permit to mitigate/eliminate specific harmonic components, which in turn can result in significant improvements of the overall performance of the machine under analysis. A practical example of the concepts detailed above is provided in Fig. 1. In Fig. 1a), a conventional symmetric slot pattern, with equal number of conductors for all of the slots and standard slot opening arrangement is sketched and compared against its corresponding modified version, with unequal number of conductors per slot and a non-standard slot opening pattern. If these groups of conductors (slots) belonged to the same phase $k, n_{k}=6 p$ for both cases indicated as "symmetric" and "modulated" in the figure. Referring to these examples, the application of (1) leads to the step-like functions shown in Fig. 1b), where $N_{k, q}$ features positive values when the convention for the current direction is assumed to be inward. The average value of the WF calculated according to (1) can be proven to be not significant, since it depends on the arbitrary choice of point $\lambda=0$; therefore, Fig. 1c) is plotted assuming that the average value is null. In the figure, 

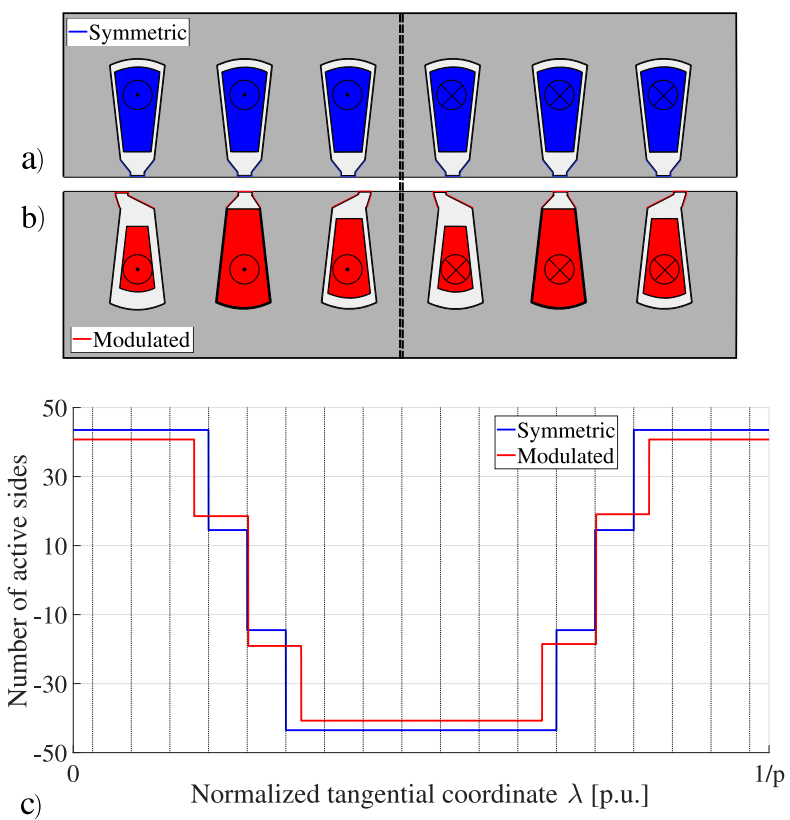

c)

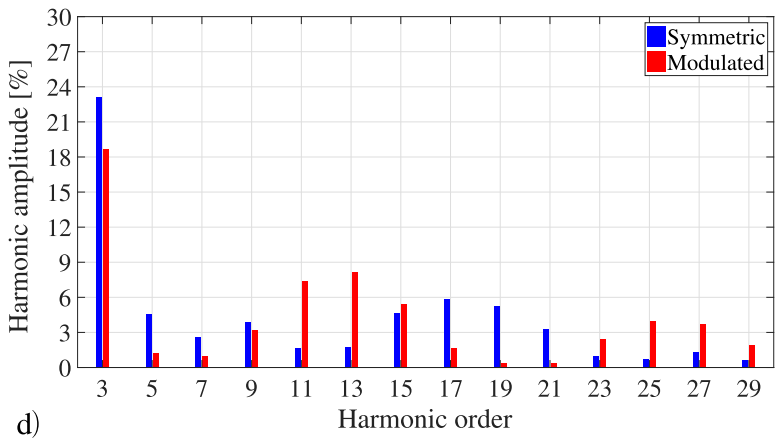

Fig. 1. a) symmetric slot opening pattern and equal number of conductors-perslot; b) modulated slot opening pattern and unequal number of conductors-perslot; comparison between symmetric and modulated c) WFs and b) amplitude of the harmonic spectra.

the WF in blue describes qualitatively the symmetric phase winding layout shown in Fig. 1a), consisting of 3 slots-perpole uniformly displaced along either the inner surface of the stator core or the outer surface of the rotor structure (i.e. a constant slot pitch is assumed). On the other hand, the red WF refers to the same generic phase when this is modulated as observed in red Fig. 1a). The red WF reflects the non-uniform slot distribution and the non-equal number of conductors-per-coil. The comparison between these WFs is also performed in terms of amplitude of the harmonic spectrum, as seen in Fig. 1d). At this stage, it is important to note only how these amplitudes can vary by exploiting the additional degrees of freedom aforementioned, without focusing on the positive implications that such alterations can lead to. However, an example of how such benefits can be achieved by exploiting asymmetries is also provided in this section. In particular, the key quantities $N_{k, q}$ and $\lambda_{k, q}(\alpha)$ involved in (6) can be opportunely selected to target (i.e. mitigate) specific MMF harmonics. The total MMF can be
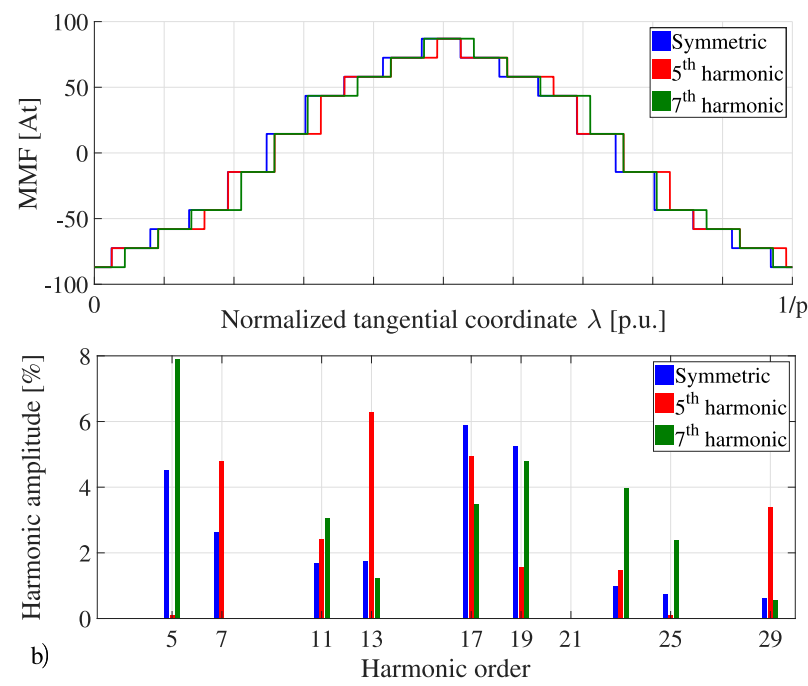

Fig. 2. Comparison between a) MMFs and b) relevant spectra resulting from symmetric (in blue) and modulated (in green and red) winding arrangements.

calculated by multiplying the vector $\bar{N}(\lambda, \alpha)$ containing all the WFs describing the machine phases by the vector $\bar{i}^{T}$ of the currents flowing inside them. Therefore, assuming a sinusoidal distribution of such currents and referring to a three-phase stator winding example, the total MMF can be determined e.g. for the symmetrical winding arrangement sketched in Fig. 1a), when this is obviously extended to a three-phase scenario. This is shown in blue in Fig. 2a). If then the slot opening positions were modified as qualitatively shown in Fig. 1b) and in such a way that all of the WFs describing the three machine phases feature identical waveforms, different MMFs are obtained. For example, the WFs can be opportunely selected to produce a MMF as that plotted in red in Fig. 2a), whose $5^{\text {th }}$ harmonic component is eliminated from the relevant spectrum of Fig. 2b). Similarly, referring to the same figures, the green MMF waveform is produced in such a way that its $7^{\text {th }}$ harmonic amplitude is removed from the spectrum. Considering the general observations carried out above, it is clear that the benefits possibly achievable by implementing asymmetric structures can be exploited and verified on real electrical machine case studies.

\section{CASE STUdy 1 - TORQue Ripple Reduction in A SQUIRREL-CAGE INDUCTION MOTOR}

As introduced in the previous sections, non-symmetric arrangements can involve different parts of the electrical machine. For example, in IMs, unconventionally displaced rotor bars and/or stator slot openings can be implemented. Also, unequal cross sections of the rotor bars and/or nonuniform number of turns-in-series-per-coil can be adopted. Although all these options can have a significant impact on the harmonic spectra of stator and/or rotor MMFs and therefore on the torque ripple, only the effects of the asymmetrical displacement of the stator slot openings are investigated in this paper. It is worth to note that the position of the slot openings 
is a key parameter to define the flux linkage with a coil loop. In fact, given a certain field map and flux lines distribution, the slot opening position discriminates whether the flux tubes link or not the active conductors located in the corresponding slot.

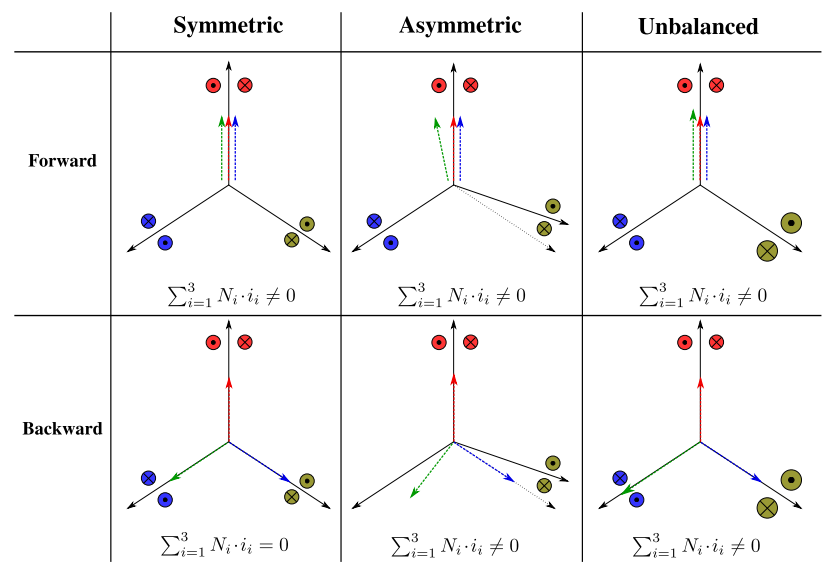

Fig. 3. Forward and backward spatial harmonics for symmetrical, asymmetrical and unbalanced winding configurations.

The stator windings of standard IMs usually consist of a three-phase, symmetrically-distributed system fed by a symmetrical set of currents. This simplified scenario can be represented by three phasors equally distributed in the space as shown in the first column of Fig. 3. In this configuration, the stator MMF can be decomposed in forward and backward rotating spatial harmonics of the first order (i.e. only the fundamental harmonic is investigated), where the backward component has zero amplitude since the sum of the three equivalent vectors is zero. However, if one of the phases is shifted as in the second column of Fig. 3, the forward component features a reduced amplitude with respect to the symmetrical case and the backward harmonic assumes a not null value. The latter component induces in the rotor cage a set of extra spatial MMFs which might deteriorate the torque ripple profile. Similar effects can be produced when considering unbalanced amplitudes of the stator MMFs, as in the case illustrated in the third column of Fig. 3. To avoid such undesired effects, the positions of the slot openings of the IM case study are modified in such a way that the stator MMF maintains the symmetrical layout schematized in the first column of Fig. 3. This constraint on the slot opening modulation was also adopted in the example provided in Fig. 2, where the slot opening positions were relocated targeting the elimination of $5^{\text {th }}$ and $7^{\text {th }}$ harmonics respectively, while limiting the impact on the fundamental harmonic.

The IM topology chosen in this paper as case study features 36 stator slots hosting a full-pitch winding and a squirrel-cage rotor comprising 28 bars. Some of these properties can be observed in Fig. 4, where a 2-D cross-section corresponding to one pole pair of the machine is illustrated. A modulated slot opening pattern is proposed to reduce the torque ripple of the IM case study. To achieve an optimal solution, genetic-

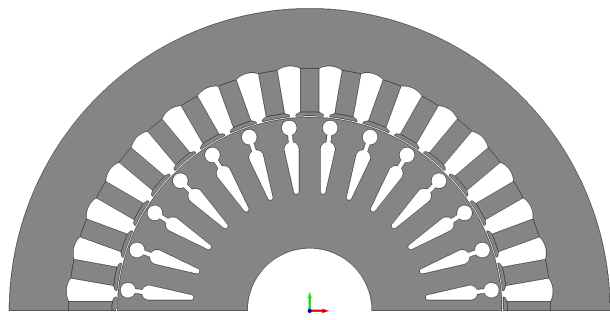

Fig. 4. 2-D cross-section of the benchmark IM.

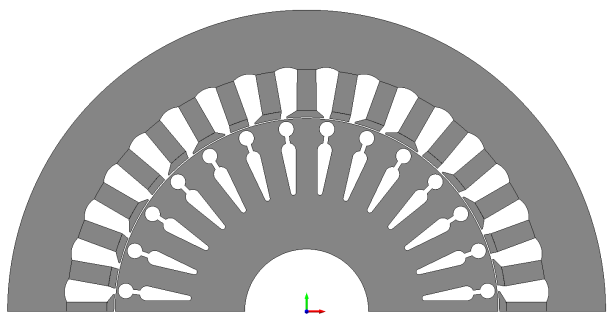

Fig. 5. 2-D cross-section of the optimal IM.
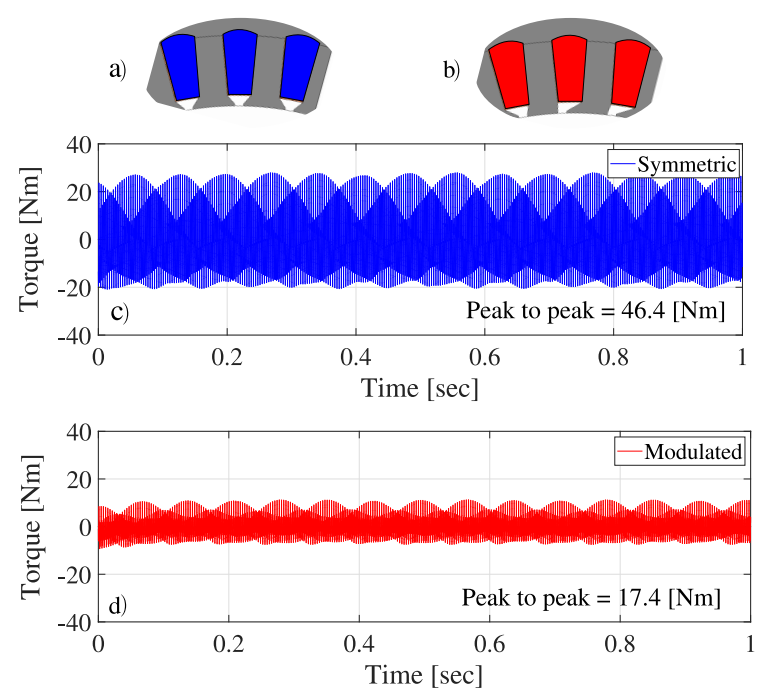

Fig. 6. Stator sectors equipped with a) the symmetric slot opening pattern and $b$ ) the modulated slot opening pattern; torque ripple featured by c) the symmetric, benchmark IM and d) the modulated, optimal IM.

algorithm-based optimizations are performed. The whole set of the machines considered during the optimization are analyzed via $\mathrm{FE}$ simulations to account for the non-linear behavior of iron materials and leakage effects in the slots. To minimize the computational burden, the number of geometrical variables is selected to ensure an odd-symmetrical structure of the IM, thus resulting in only 3 slot openings per-pole-per-phase to be optimized. The total number of solutions investigated is equal to 1500 , subdivided in 30 populations comprising 50 individuals each. The multi-objective optimization tool based on the genetic algorithm named NSGA-II [18] implemented within the MATLAB optimization toolbox is used to find the optimal stator topology. The objective functions are set in 
such a way to minimize the torque ripple while keeping the average torque the same as in the baseline machine. One of the Pareto front solutions is selected for the sake of comparison against the benchmark machine. A 2-D cross-section of this optimal machine is reported in Fig. 5. A comparison between benchmark (indicated as symmetric) and optimal (indicated as modulated) stator sectors is observed in Fig. 6a) and Fig. 6b). In Fig. 6c), the output torque profile (subtracted by its average value) of the original machine is presented. The stator portion of the benchmark machine (indicated as symmetric in the legend) under analysis is sketched in Fig. 6c). Fig. 6d) shows the output torque profile of the modulated IM. It can be noticed from the comparison that the optimal solution achieves a $63 \%$ peak-to-peak torque reduction, confirming the potentiality of the proposed methodology.

\section{CASE Study 2 - No-LoAd Voltage THD Reduction IN A SALIENT-POLE SYNCHRONOUS GENERATOR}

The damper cage of SGs is being widely studied and have been proven to play an important role on the mechanical dynamic transient response as well as in steady-state performance of the machine [19]. To further confirm the potential of the method presented in this paper, a re-positioning of the damping bars equipping a SG case study is considered aiming to improve cage losses and output voltage THD.

The alternator under investigation features 48 stator slots hosting a 3-phase winding with 4 slots-per-pole-phase and 4 rotor salient poles with 6 bars-per-pole. A 2-D cross-section corresponding to one pole pair of this machine is reported in Fig. 7. To differentiate with respect to the IM case, where an odd-symmetric structure was maintained even onto the optimal machine, the positions of the bars embedded inside two adjacent poles are tailored for the SG case study. Hence, a symmetric periodic configuration spanning 2 pole pitches is obtained, thus resulting in an increased number of degrees of freedom (i.e. the positions of 12 bars) compared to the case when only the bars on one salient pole are re-positioned (i.e. the positions of 6 bars).

To achieve an optimal solution which addresses both bar losses and voltage THD, a genetic-algorithm-based optimization process is adopted also for such case study. The whole set of the machines considered during the optimization are analyzed via transient-with-motion FE evaluations. The total number of solutions investigated is equal to 1225 , subdivided in 25 populations comprising 50 individuals each. The multiobjective genetic algorithm named MOGA-II [20] implemented within the software ModeFrontier by Esteco is used to find the optimal damper cage topology. The main objective function is the no-load voltage THD. This is set to be lower than $3 \%$. Considering that the asymmetrical damper cage patterns can have an impact on the amplitudes of the currents induced inside the bars, also the total bar loss is an output optimization variable. A complete description of the optimization procedure and the relevant results are provided in [13]. Given the goals of this paper, only the optimal machine

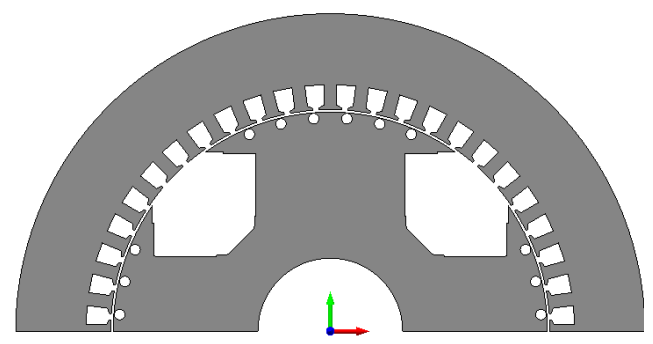

Fig. 7. 2-D cross-section of the benchmark SG.

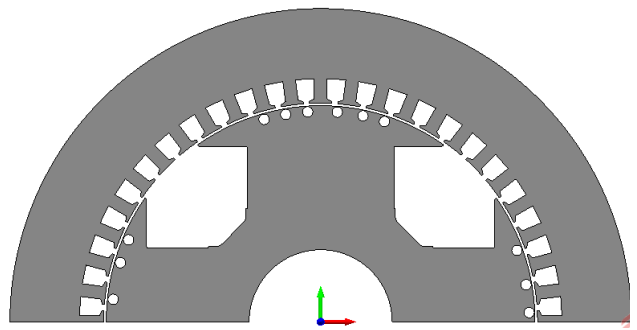

Fig. 8. 2-D cross-section of the optimal SG.
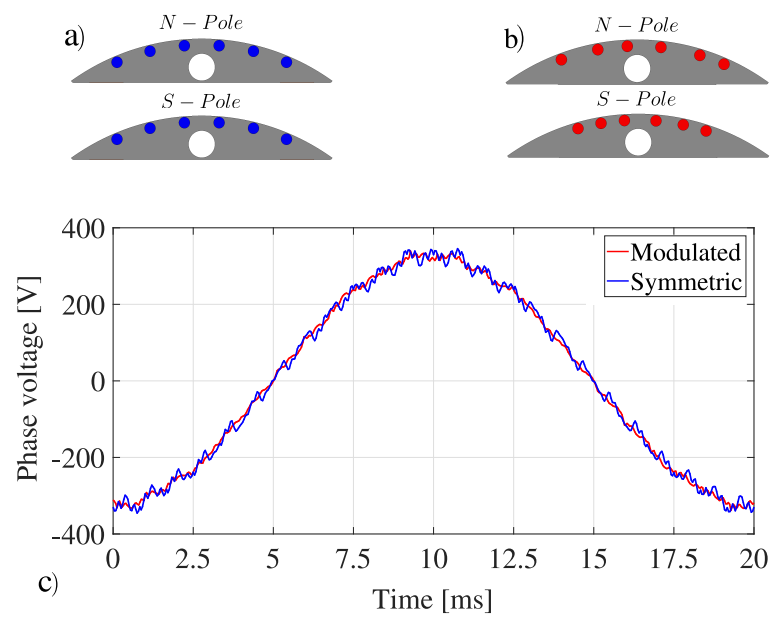

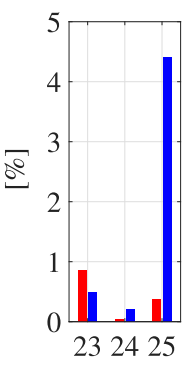

d)

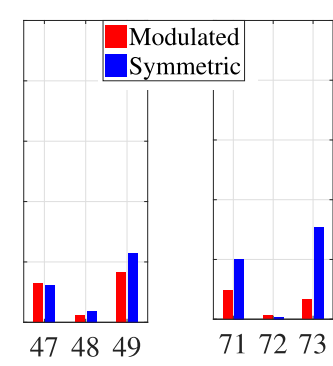

Harmonic order

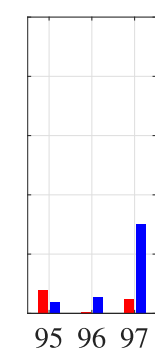

959697
Fig. 9. Salient poles equipped with a) the symmetric damper cage and b) the modulated damper cage; comparison between c) no-load phase voltage waveforms and d) relevant harmonic spectra.

is presented and compared against the benchmark SG. A 2-D cross section of the machine comprising the optimal damper cage is shown in Fig. 8. A comparison between the 
benchmark (indicated as symmetric) and the optimal (indicated as modulated) damper cage topologies is also observed in Fig. 9a) and Fig. 9b). The FE-evaluated no-load voltages of the benchmark and optimal SGs are compared in Fig. 9c), highlighting the waveforms' quality improvement due to the application of the asymmetrical bars' pattern. In Fig. 9d) the amplitude of the harmonic spectra of such waveforms are compared. Here, only the slotting harmonics groups are highlighted, showing the effectiveness of the modulated concept. Overall, the optimal damper cage permits to achieve a THD improvement of $54 \%$, i.e. from $5.58 \%$ to $2.56 \%$. As reported in [13], this achievement was also confirmed by experimental measurements carried out on a 400-kVA prototype built for validation purposes. A no-load line-to-line voltage THD equal to $2.29 \%$ was recorded during testing.

\section{CONCLUSIONS}

This work proposes asymmetrical windings' and core structures' patterns as means to address challenges commonly investigated in electrical machines. First, the potential of exploiting the degrees of freedom associated to such unconventional configurations was emphasized through a mathematical survey aimed at finding a compact expression of the Fouries series harmonic amplitudes of the winding functions associated to any generic machine phase. The latter is to be intended in a broad sense, thus including all the types of rotor and stator windings employed in electrical machines. Although in this paper focus was given to the windings and their relevant describing functions, the whole reasoning may be extended to additional machine design variables and parameters. Referring to the analytical findings and keeping a general approach, the effects of some asymmetric pattern examples on winding functions and magneto-motive forces were studied and highlighted. Finally, two case studies were analysed to prove the perceived benefits:

1) the peak-to-peak torque of an IM was reduced by $63 \%$ by repositioning only 3 slot openings per-pole-per-phase, without compromising the average output torque;

2) the no-voltage THD of a salient-pole SG was improved by $54 \%$ by tailoring the positions of the bars embedded onto 2 adjacent poles, without deteriorating the rotor bar losses.

The design methodologies proposed in this paper could come at the cost of slightly complicating the associated manufacturing processes. However, their exploitation could permit to remove the need for disruptive techniques such as stator and/or rotor skewing, short pitched windings, etc. used for similar purposes. This could in turn result in game-changing opportunities for all the types of electrical machines and, in particular, even in a revamping of the well-consolidated IMs' and SGs' design processes.

\section{ACKNOWLEDGMENT}

The financial support from ABB Corporate Research in Västerảs for this research is gratefully acknowledged.

\section{REFERENCES}

[1] T. J. Juha Pyrhonen and V. Hrabovcov, Design of Rotating Electrical Machines. John Wiley \& Sons, Ltd, 20008.

[2] P. L. Alger, Induction machines, Gordon and breach science, Eds., 1970.

[3] A. F. C. Kingsley and S. D.Umans, Electric machinery. Mc Graw Hill, 2003.

[4] A. Marfoli, G. Sala, L. Papini, P. Bolognesi, and C. Gerada, "Torque ripple investigation in squirrel cage induction machines," in 2019 IEEE International Electric Machines Drives Conference (IEMDC), May 2019, pp. 140-146.

[5] A. Al-Timimy, P. Giangrande, M. Degano, Z. Xu, M. Galea, C. Gerada, G. Lo Calzo, H. Zhang, and L. Xia, "Design and losses analysis of a high power density machine for flooded pump applications," IEEE Transactions on Industry Applications, vol. 54, no. 4, pp. 3260-3270, July 2018.

[6] F. J. T. E. Ferreira, G. Baoming, and A. T. de Almeida, "Reliability and operation of high-efficiency induction motors," IEEE Transactions on Industry Applications, vol. 52, no. 6, pp. 4628-4637, Nov 2016.

[7] V. Madonna, P. Giangrande, L. Lusuardi, A. Cavallini, C. Gerada, and M. Galea, "Thermal overload and insulation aging of short duty cycle, aerospace motors," IEEE Transactions on Industrial Electronics, pp. 1$1,2019$.

[8] S. X. Duarte and N. Kagan, "A power-quality index to assess the impact of voltage harmonic distortions and unbalance to three-phase induction motors," IEEE Transactions on Power Delivery, vol. 25, no. 3, pp. 18461854, July 2010.

[9] D. Fallows, S. Nuzzo, A. Costabeber, and M. Galea, "Harmonic reduction methods for electrical generation: a review," IET Generation, Transmission Distribution, vol. 12, no. 13, pp. 3107-3113, 2018.

[10] S. Nuzzo, M. Degano, M. Galea, C. Gerada, D. Gerada, and N. Brown, "Improved damper cage design for salient-pole synchronous generators," IEEE Transactions on Industrial Electronics, vol. 64, no. 3, pp. 19581970, March 2017.

[11] A. Tessarolo, "A quadratic-programming approach to the design optimization of fractional-slot concentrated windings for surface permanentmagnet machines," IEEE Transactions on Energy Conversion, vol. 33, no. 1, pp. 442-452, March 2018.

[12] D. A. Kocabas, "Novel winding and core design for maximum reduction of harmonic magnetomotive force in ac motors," IEEE Transactions on Magnetics, vol. 45, no. 2, pp. 735-746, Feb 2009.

[13] S. Nuzzo, P. Bolognesi, G. Vakil, D. Fallows, C. Gerada, N. L. Brown, and M. Galea, "A methodology to remove stator skew in small-medium size synchronous generators via innovative damper cage designs," IEEE Transactions on Industrial Electronics, pp. 1-1, 2018.

[14] N. Bianchi, S. Bolognani, D. Bon, and M. Dai Pre, "Rotor flux-barrier design for torque ripple reduction in synchronous reluctance and pmassisted synchronous reluctance motors," IEEE Transactions on Industry Applications, vol. 45, no. 3, pp. 921-928, May 2009.

[15] M. Sanada, K. Hiramoto, S. Morimoto, and Y. Takeda, "Torque ripple improvement for synchronous reluctance motor using an asymmetric flux barrier arrangement," IEEE Transactions on Industry Applications, vol. 40, no. 4, pp. 1076-1082, July 2004.

[16] J. Wanjiku, M. A. Khan, P. S. Barendse, and P. Pillay, "Influence of slot openings and tooth profile on cogging torque in axial-flux pm machines," IEEE Transactions on Industrial Electronics, vol. 62, no. 12, pp. 75787589, Dec 2015.

[17] A. Marfoli, P. Bolognesi, L. Papini, and C. Gerada, "Mid-complexity circuital model of induction motor with rotor cage: A numerical resolution," in 2018 XIII International Conference on Electrical Machines (ICEM), Sept 2018, pp. 277-283.

[18] K. Deb, Multi-Objective Optimization using Evolutionary Algorithms. John Wiley \& Sons ISBN 047187339.

[19] S. Nuzzo, P. Bolognesi, C. Gerada, and M. Galea, "Simplified damper cage circuital model and fast analytical-numerical approach for the analysis of synchronous generators," IEEE Transactions on Industrial Electronics, pp. 1-1, 2018.

[20] S. Poles, "Moga-ii an improved multi-objective genetic algorithm," Esteco, Trieste, Italy, 2003, Tech. Rep. 2003-006. 